\title{
NARROW-BAND PHOTOMETRY AND THE STELLAR POPULATIONS IN ELLIPTICAL GALAXIES*
}

\author{
KARL D. RAKOS \\ Institute for Astronomy, University of Vienna, Austria \\ TOBIAS J. KREIDL \\ Lowell Observatory, Flagstaff, Arizona, U.S.A. \\ and \\ JAMES M. SCHOMBERT \\ Dept. of Astronomy, University of Michigan, Ann Arbor, Michigan, U.S.A.
}

\begin{abstract}
The modified Strömgren $u v b y$ filter system, which has been used to investigate the spectrophotometric evolution of elliptical galaxies, is also found to be a powerful tool for the determination of stellar parameters in elliptical galaxies, especially metal content and the stellar type of the dominant population. We present an introduction to the behaviour of the filter system based on comparison to stellar libraries and then explore the results with respect to composite systems: globular clusters, spirals, and ellipticals.
\end{abstract}

\section{Introduction}

In the last three papers (Fiala et al., 1986; Rakos et al., 1988, 1989), we have presented the details of our modified Strömgren filter system (called $u z, v z, b z, y z$ ), developed methods of discriminating between stars and peculiar objects, developed methods of identifying cluster membership and Hubble-type and discussed interpretation of our results with respect to colour evolution in distant clusters. Comparison to spectro-evolution models suggested a blueward shift in colours as a function of redshift to the degree not expected from residual star formation, yet not predicted by the models. Since the models are produced from single metallicity populations, the possibility was considered that the deviant colours are due to changes in the global metallicity values with time (i.e., chemical evolution). In Paper III, we presented an introduction to the use of the narrow-band system as a metallicity probe and delivered the equations relinking our $u z$, $v z, b z, y z$ system to the original Strömgren system in order to investigate the observed metallicities and stellar content indices of ellipticals as compared to model atmospheres, metallicity standards and galactic globulars. In this paper, we will expand on these correlations and demonstrate their applicability with respect to a few specific examples from data in the literature.

\footnotetext{
* Paper presented at the 11th European Regional Astronomical Meetings of the IAU on 'New Windows to the Universe', held 3-8 July, 1989, Tenerife, Canary Islands, Spain.
} 


\section{Calibration of Two-Colour Diagrams}

We have shown in Paper III that the position of stellar system in the two-colour $(v z-y z),(b z-y z)$ diagram is directly dependent on the mean metal content of the system and independent of the mean surface gravity due to the low-temperature stellar range dominant in ellipticals. We have also derived a relationship between $[\mathrm{Fe} / \mathrm{H}]$, the metallicity colour $(v z-y z)$ and the continuum colour $(b z-y z)$ of the form

$$
[\mathrm{Fe} / \mathrm{H}]=\frac{0.334+2.1(v z-y z)-5.651(b z-y z)}{0.157+0.311(b z-y z)}
$$

for the range $-2.1<[\mathrm{Fe} / \mathrm{H}]<0$ and $(b z-y z)>0.14$.

This relationship also holds for composite stellar systems with a single type of colour distribution of stars within a magnitude in colour difference. On the other hand, a bimodal distribution of colours would mimic lower metallicity, e.g., the presence of significant number of blue horizontal branch stars.

The next step in the application of the narrow-band photometry to the elliptical galaxies is the estimation of the dominant population in the bandpass of observations (in this case near-UV and blue luminosities). For this reason, we have examined two possible colour-colour diagrams, namely $(u z-y z)$ vs $(v z-y z)$ and $(u z-v z)$ vs $(v z-b z)$. It can be shown that for the range $(b z-y z)>0.14$, there are well-defined relations for Main Sequence and giant stellar tracks. For 45 Main-Sequence stars compiled from Crawford and Barnes (1970) and Hauck and Mermilliod (1980) with $[\mathrm{Fe} / \mathrm{H}]=0$ we obtain

$$
\begin{array}{ll}
(u z-y z)=0.352+1.320(v z-y z) & (\mathrm{MS} \text { stars, }[\mathrm{Fe} / \mathrm{H}]=0), \\
(u z-v z)=0.275+0.529(v z-b z) & \text { (MS stars, }[\mathrm{Fe} / \mathrm{H}]=0),
\end{array}
$$

with a correlation coefficient of $R=0.98$. For 32 Main-Sequence stars of extreme Population II with a mean value of $[\mathrm{Fe} / \mathrm{H}] \simeq-1.5$, compiled from Olsen (1983), Francois (1986), Magain (1987), and Toukin et al. (1985), there is a fair relationship

$$
\begin{array}{ll}
(u z-y z)=0.329+1.294(v z-y z) & (\mathrm{MS} \text { stars, }[\mathrm{Fe} / \mathrm{H}]=-1.5), \\
(u z-v z)=0.373+0.490(v z-b z) & (\mathrm{MS} \text { stars, }[\mathrm{Fe} / \mathrm{H}]=-1.5)
\end{array}
$$

with a correlation coefficient of $R=0.87$. A similar procedure for giants of the solar metal content, totalling 30 stars from Jacoby et al. (1984) and Crawford and Barnes (1970) delivers

$$
\begin{array}{ll}
(u z-y z)=0.634+1.310(v z-y z) & (\text { giant stars, }[\mathrm{Fe} / \mathrm{H}]=0), \\
(u z-v z)=0.673+0.481(v z-b z) & (\text { giant stars, }[\mathrm{Fe} / \mathrm{H}]=0),
\end{array}
$$

with a correlation coefficient of $R=0.96$. For 47 giants with a mean value of $[\mathrm{Fe} / \mathrm{H}]=-2.1$ compiled from Luck and Bond (1985) and Gratton and Sneden (1988) we have

$$
(u z-y z)=-0.217+1.651(v z-y z) \quad(\text { giant stars, }[\mathrm{Fe} / \mathrm{H}]=-2)
$$




$$
(u z-v z)=0.366+1.122(v z-b z) \quad(\text { giant stars, }[\mathrm{Fe} / \mathrm{H}]=-2),
$$

with a correlation coefficient of $R=0.99$. All these relations are based on reddening-free stars and are shown in Figurs 1 and 2. Reddening vectors for both diagrams can be also drawn with the relations

$$
\begin{aligned}
& E(u z-y z)=1.52 E(y z-y z), \\
& E(u z-v z)=1.29 E(v z-b z),
\end{aligned}
$$

derived in Paper III. Unfortunately, there are no model atmospheres calculated for late spectral types to test these equations on theoretical stellar models. However, for the limited accuracy of narrow-band photometry of elliptical galaxies in distant clusters (typical errors of $\sigma=0.05 \mathrm{mag}$ ), our equations are more than adequate.

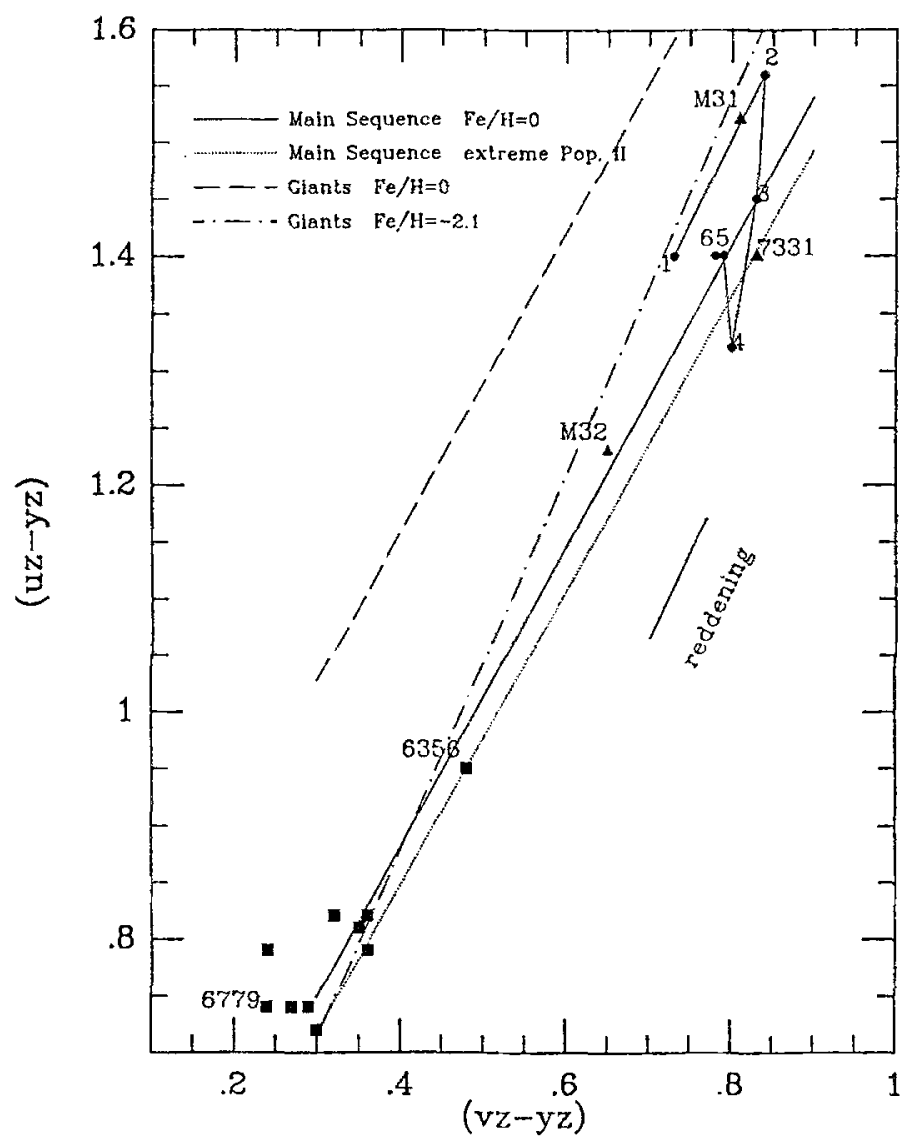

Fig. 1. The $(u z-y z),(v z-y z)$ diagram. The solid line represents the Main-Sequence $[\mathrm{Fe} / \mathrm{H}]=0$ stars; the dotted line represents Main-Sequence extreme Population II stars. The broken line shows the position of giants with $[\mathrm{Fe} / \mathrm{H}]=0$ and the line containing dashes and dots indicates the position of metal-poor $([\mathrm{Fe} / \mathrm{H}]=-2.1)$ giants. Also plotted are objects of interest. The objects labeled with $1,2,3, \ldots, 6$ are elliptical galaxies in clusters with increasing redshift as stated in Paper III. 


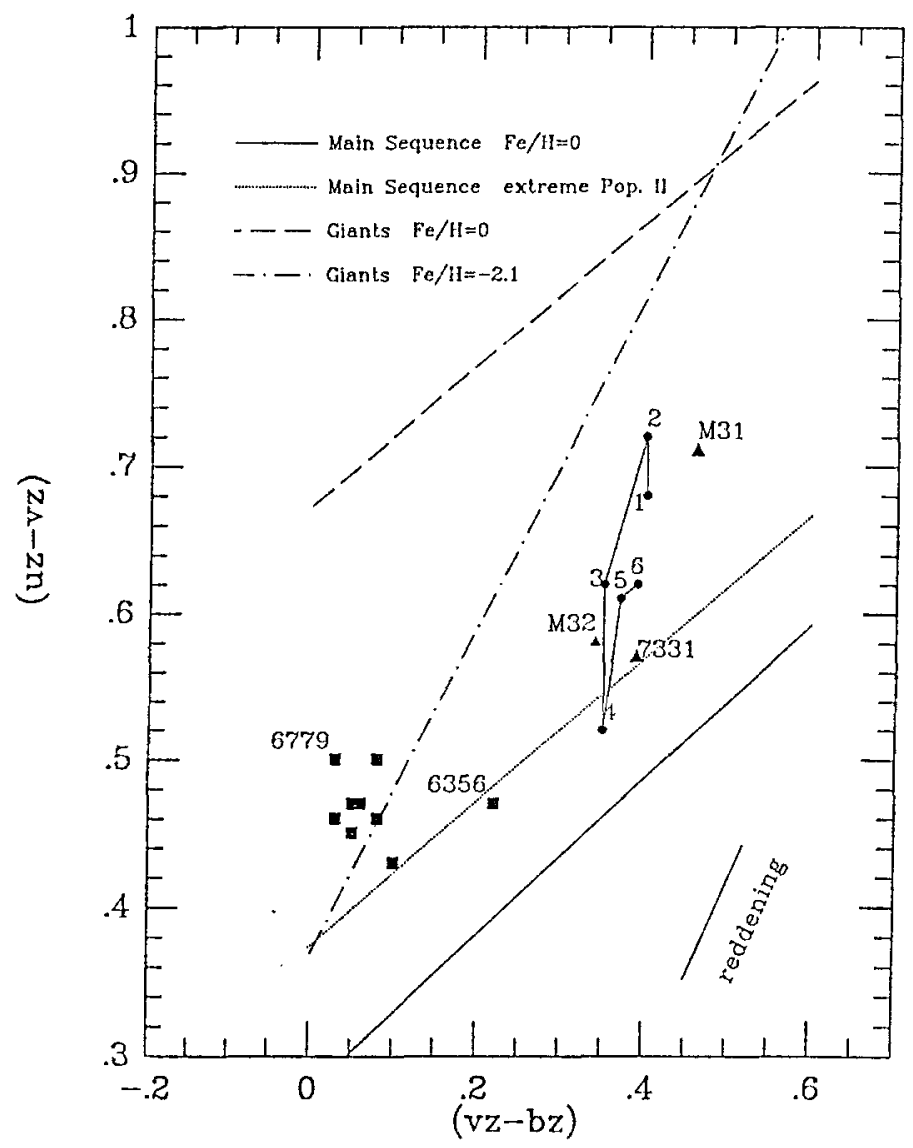

Fig. 2. The $(u z-v z),(v z-b z)$ diagram. Lines and symbols are the same as in Figure 1.

Examination of Figures 1 and 2 shows the difficulty in deriving population parameters free of metallicity effects. Basically, as outlined in Paper III, the $b z$ and $y z$ filters form a metal and surface gravity free index of continuum colours. The $v z$ filter samples a metal line region of the spectrum and, thus, the $(v z-y z),(b z-y z)$ diagram is a populationtype independent measure of $[\mathrm{Fe} / \mathrm{H}]$. The $u z$ samples the region dominated by surface gravity effects and, therefore, a measure of the integrated population type (dwarf vs giant stars); however, by definition, this region is also sensitive to metallicity and this produces a coupling in any $u z$ colour vs continuum colour diagram between $[\mathrm{Fe} / \mathrm{H}]$ and population type. The solution is to use a population plus metallicity colour vs a metallicity colour of which the two examined above and shown in Figure 1 and 2 are $(u z-y z)$, $(v z-y z)$ and $(u z-v z),(v z-b z)$. The main advantage to the $(u z-y z),(v z-y z)$ diagram is that the reddening vector is parallel to the tracks and, therefore, the effects of gas and dust on the indices is minimal. However, the tracks are strongly coupled when the $u z$ and $v z$ filters are used in opposition. The $(u z-v z),(v z-b z)$ is more strongly effected by reddening, but the population tracks are less coupled and the dominant population 
type is more easily discernable. This diagram is the tool of choice for ellipticals, which are free of gas and dust.

\section{Application to the Elliptical Galaxies}

In order to demonstrate the usefulness of Figures 1 and 2 for the estimation of the dominant stellar population, we have convolved the Strömgren or narrow-band colours from Faber (1973) and Hunter (1982) to our system (see Tables 2, 4, and 14 from Paper III) and are plotted in Figures 1 and 2. In general, the globular clusters are characterized by metal-poor giant stars, confirming what has been known for years in integrated light and spectroscopic studies. The galaxies M32 (a compact elliptical), M31 and NGC 7331 ( $\mathrm{Sb}$ spirals) are shown. Also shown are the elliptical tracks from Paper III with point 1 being the mean colours of present-day ellipticals. These examples are discussed below.

- The two very distinctive globular clusters are NGC 6365, a metal-rich cluster with $[\mathrm{Fe} / \mathrm{H}]=-0.62$ and $\mathrm{NGC} \mathrm{6779}$, a metal-poor cluster with $[\mathrm{Fe} / \mathrm{H}]=-1.94$. NGC 6365 is located in Figure 1 near the lines for the Main-Sequence stars and low-metal content giants. Because the cluster is metal-rich, it cannot have a large number of giants (of low-metal content). In Figure 2, we see that the cluster has many more Main-Sequence stars than the other globular clusters in the diagram, especially NGC 6779. NGC 6779 seems to be composed almost entirely from low-metal content giants. If one assumed that NGC 6779 were additionally reddened, than a Population II Main-Sequence stars should be added.

- M32 is a E2 galaxy of solar metallicity type. Figure 1 predicts about $90 \%$ of light is radiated from Main-Sequence stars. In Figure 2, the Main Sequence contributes only $60 \%$ of the light. The difference is primarily due to the internal errors in the data and the accuracy of the method used.

- NGC 7331 is a Sb I-II metal-poor galaxy with $[\mathrm{Fe} / \mathrm{H}]=-1.39$. In Figures 1 and 2, we would estimate that the flux from the galaxy mainly belongs to the MainSequence extreme Population II stars. Internal reddening is always an unknown for optical colours of spirals; however, the integrated light from this galaxy is primarily from the bulge which is dust free. This is confirmed by the similar positions in both Figures 1 and 2.

- M31 a Sb I-II galaxy with solar metal content shows in both figures about $50 \%$ light from the Main Sequence and 50\% from giants. Again the light is based on aperture values which are bulge dominated and little effected by reddening.

The mean value of field elliptical galaxies (labeled as 1 in Figures 1 and 2) is very similar to that of M31. For ellipticals with increasing redshift (represented by the numbered sequence), we see an increasing contribution of Main-Sequence low-metal population stars. For $z=0.39$, nearly all stars belong to the Main Sequence. A similar change between giants and Main Sequence has been proposed in the spectrophotometric evolution of galaxies by Guiderdoni and Rocca-Volmerange (1987) in their Figures 6(a) and $6(\mathrm{~b})$. The time-scale of the calculated change in the luminosity fraction of giants and 
Main-Sequence stars seems to be in disagreement with our results, but to resolve this problem, we require a statistically significant number of observations, as well as better theoretical models which include changing metallicity as a function of time.

\section{Conclusions}

Narrow-band photometry allows one to follow the change of the mean metallicity and the luminosity fraction of giants vs Main Sequence for large redshifts up to about $z=0.8$ or into the past nearly as far as the half age of the Universe. In agreement with Rose (1985), we find a strong dwarf dominance in the integrated light of galaxies determined from blue optical colours. Unlike optical to IR colours, the small mixture of ages within a galaxy has little effect on our filters since the close spacing of the bandpasses emphasizes the turnoff point and base of the giant branch rather than the tip of the giant branch. Uniqueness problems to the stellar populations can be iterated with a statistically significant number of high-quality observations combined with an improved theory and models for the spectral evolution of galaxies.

\section{Acknowledgement}

We gratefully acknowledge the financial support from the Austrian 'Fonds zur Förderung der Wissenschaftlichen Forschung'.

\section{References}

Crawford, D. L. and Barnes, J. V.: 1970, Astron. J. 75, 978.

Faber, S. M.: 1973, Astrophys. J. 179, 731.

Fiala, N., Rakos, K., and Stockton, A.: 1986, Publ. Astron. Soc. Pacific 98, 70.

Francois, P.: 1986, Astron. Astrophys. 160, 264.

Gratton, R. G. and Sneden, C.: 1988, Astron. Astrophys. 204, 193.

Guiderdoni, B. and Rocca-Volmerange, B.: 1987, Astron. Astrophys. 186, 1.

Hauck, B. and Mermilliod, M.: 1980, Astron. Astrophys, Suppl. 40, 1.

Hunter, D. A., Gallagher, J. P., and Rautenkranz, D.: 1982, Astrophys. J. Suppl. 49, 53.

Jacoby, G. H., Hunter, D. A., and Christian, C. A.: 1984, Astrophys. J. Suppl. 56, 257.

Luck, R. E. and Bond, H. E.: 1985, Astrophys. J. 292, 559.

Magain, P.: 1987, Astron. Astrophys. 179, 176.

Olsen, H.: 1983, Astron. Astrophys. Suppl. 54, 55.

Rakos, K. D., Fiala, N., and Schombert, J. M.: 1988, Astrophys. J. 328, 463.

Rakos, K. D., Schombert, J. M., and Kreidl, T. J.: 1989, Astrophys. J. (submitted).

Rose, J. A.: 1985, Astron. J. 90, 1927.

Toukin, J., Lambert, D. L., and Balachandran, S.: 1985, Astrophys. J. 290, 289. 